PROCEEDINGS OF THE

AMERICAN MATHEMATICAL SOCIETY

Volume 132, Number 7, Pages 1891-1898

S 0002-9939(03)07292-7

Article electronically published on December 18, 2003

\title{
SUPERDECOMPOSABLE PURE-INJECTIVE MODULES EXIST OVER SOME STRING ALGEBRAS
}

\author{
GENA PUNINSKI \\ (Communicated by Martin Lorenz) \\ ABSTRACT. We prove that over every non-domestic string algebra over a count- \\ able field there exists a superdecomposable pure-injective module.
}

\section{INTRODUCTION}

There is a well-known dichotomy for the behavior of a finite-dimensional algebra $A$ over a field $\mathbb{k}$. Roughly speaking, $A$ is tame if a description of all finitedimensional $A$-modules is available, otherwise $A$ is wild. This definition can be made precise, and then Drozd's theorem states (at least for an algebraically closed $\mathbb{k}$ ) that every finite-dimensional algebra is either tame or wild but not both.

Unfortunately, the usual definition of tameness and wildness refers to some infinite-dimensional $A$-modules (what Ringel [10] describes as an "external structure"). So it would be nice to find one appealing only to finite-dimensional representations. It has been conjectured by Prest [6, Ch. 13] (see also [10, p. 38] and a discussion in [5, p. 219]) that $A$ is tame if and only if $A$ does not possess a superdecomposable (i.e., without indecomposable direct summands) pure-injective module. This means just that every direct product of indecomposable finite-dimensional $A$ modules contains an indecomposable direct summand.

In this paper we refute this conjecture by proving that over an arbitrary nondomestic string algebra over a countable field there exists a superdecomposable pure-injective module. This class of algebras is well known to be tame and includes among others the Gelfand-Ponomarev algebras as well as the dihedral algebras. So it seems now that the classification of pure-injective modules over a non-domestic string algebra (just slightly touched on by Baratella and Prest [1]) is a more challenging problem than previously believed.

Is countability of $\mathbb{k}$ necessary in the above result? In fact, our main result does not appeal to any countability assumption: we prove that the lattice of all pp-formulae over any non-domestic string algebra does not have width. But to extract a superdecomposable pure-injective module from this we need an ingenious construction of Ziegler [12] that seems to work only if $\mathbb{k}$ is countable.

Received by the editors December 9, 2001 and, in revised form, March 19, 2003.

2000 Mathematics Subject Classification. Primary 16G20, 16D50.

Key words and phrases. Pure-injective module, string algebra, superdecomposable module.

This paper was written while the author visited the University of Manchester and was supported by EPSRC grant GR/R44942/01. He would like to thank the University for their kind hospitality. 
Note that the existence of a superdecomposable pure-injective module over a Gelfand-Ponomarev algebra was posed as a problem in Jensen and Lenzing [5] (see Remark 8.72 and Problem 13.28). So we give a partial, i.e., over a countable field, answer to this question. The reader may also consult $[5]$ to see how to construct a superdecomposable pure-injective module over many (conjecturally all) wild finitedimensional algebras.

All the machinery used in the proofs is quite well known. Prest [7] was the first to notice that over the dihedral (and many similar) algebras there exists a densely ordered chain of morphisms between string modules. In other words, the lattice of all pp-formulae over these algebras does not have $m$-dimension. This result (with a similar proof) was extended by Schröer [11 to an arbitrary non-domestic string algebra.

It is also well known (see Ringel $[8]$ for a detailed explanation) that over a dihedral algebra there are two natural chains of proper morphisms between indecomposable finite-dimensional modules. All we have noticed is that the (distributive) lattice generated by these two chains is generated freely; therefore, its width is undefined.

\section{Preliminaries}

Quite a few model-theoretic terms, which appear in what follows, can be found in [6]. Otherwise, as explained in [7], one could always replace the term "pp-formula" by "pointed finitely presented module", and the term "implication between ppformulae" by "morphism between pointed modules". All the modules in the sequel will be left modules.

Let $A$ be a finite-dimensional algebra given by a quiver with monomial relations. For an arrow $\alpha$, we will denote by $s(\alpha)$ the starting point of $\alpha$ and by $e(\alpha)$ the ending point of $\alpha$. Also, for every arrow $\alpha$, we consider its formal inverse $\alpha^{-1}$ as an arrow going into an opposite direction. Thus $e\left(\alpha^{-1}\right)=s(\alpha)$ and $s\left(\alpha^{-1}\right)=e(\alpha)$.

$A$ is said to be a string algebra if the following holds true: 1) every vertex is a starting point for at most two arrows and the ending point for at most two arrows; 2 ) given an arrow $\alpha$, there is at most one arrow $\beta$ such that $e(\beta)=s(\alpha)$, and the composition $\alpha \beta$ is not a relation in $A$ (i.e., nonzero in $A$ ); 3 ) given an arrow $\alpha$, there is at most one arrow $\gamma$ such that $e(\alpha)=s(\gamma)$, and the composition $\gamma \alpha$ is not a relation in $A$.

For instance, the Gelfand-Ponomarev algebra $G_{n, m}$ is the path algebra of the quiver

with relations $\alpha \beta=\beta \alpha=\alpha^{n}=\beta^{m}=0$. It is well known that $G_{n, m}$ is tame non-domestic if $m+n \geq 5$.

Let $A$ be a string algebra. A string $C$ over $A$ is a sequence $c_{1} \ldots c_{n}$ with the following properties: 1) for every $i$, either $c_{i}=\alpha$ is a (direct) arrow, or $c_{i}=\alpha^{-1}$ is an inverse arrow; 2) $s\left(c_{i}\right)=e\left(c_{i+1}\right)$ for every $1 \leq i \leq n-1$;3) $c_{i} \neq c_{i+1}^{-1}$ for every $1 \leq i \leq n-1$; 4) neither $c_{i} \ldots c_{i+t}$ (direct arrows) nor $c_{i+t}^{-1} \ldots c_{i}^{-1}$ (inverse arrows) is a relation in $A$ for $1 \leq i<i+t \leq n$.

Given a string $C=c_{1} \ldots c_{n}$, we define a string module $M(C)$ in the following way. The $\mathbb{k}$-basis for $M(C)$ is given by vectors $z_{0}, \ldots, z_{n}$. If $c_{i}=\alpha$ is direct, then set $\alpha z_{i}=z_{i-1}$, and if $c_{i}=\beta^{-1}$ is inverse, then put $\beta z_{i-1}=z_{i}$. All the remaining actions are defined to be zero. Following [11] we draw direct arrows from upper right to lower left and inverse arrows from upper left to lower down. 
For instance,

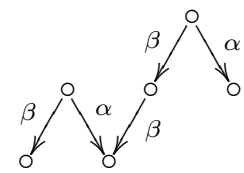

is a string module over $G_{2,3}$ corresponding to the string $\beta \alpha^{-1} \beta^{2} \alpha^{-1}$.

By [2] all string modules over a string algebra are indecomposable.

Let $C=c_{1} \ldots c_{n}, n \geq 1$ be a string. What are the possible ways to extend this string to a string $c_{1} \ldots c_{n} c_{n+1}$ ? Suppose that $c_{n}$ is a direct letter $\alpha$. If $c_{n+1}$ is a direct letter $\beta$, then $\beta$ ends in the vertex where $\alpha$ starts. Since $\alpha \beta$ is a string, there is only one possibility for $\beta$ (such that $\alpha \beta$ is not a relation in $A$ ). On the other hand, if $c_{n+1}$ is an inverse letter $\gamma^{-1}$, then $\alpha$ and $\gamma$ start at the same vertex (and $\alpha \neq \gamma$ since $C c_{n+1}$ is a string). Since there are at most two arrows starting in the given vertex, $\gamma$ is uniquely defined. Moreover, if both $\beta$ and $\gamma$ are defined, then $\alpha \beta \neq 0$ implies $\gamma \beta=0$.

Now we define a (linear) order $<$ on the set of strings with the same first (direct) letter. For strings $B$ and $C$ we put $B<C$ if one of the following holds true: 1) $B \gamma^{-1} D=C$ for some $\gamma, D$; 2) $B=C \beta E$ for some $\beta, E$; or 3$) B=B^{\prime} \beta F$, $C=B^{\prime} \gamma^{-1} G$ for some $B^{\prime}, F, \gamma$ and $G$. Thus, to compare two strings we look at their common initial part (by assumption, there is at least one letter in this part), and compare letters following this part.

Note that, if $B<C$ by 1 ), then $B<C S$ for arbitrary $S$ (such that $C S$ is a string). Similarly, if $B<C$ by 2), then $B T<C$ for any $T$. Finally, if $B<C$ by $3)$, then $B U<C V$ for all $U$ and $V$.

Lemma 2.1. Let $B<C$ be strings with first letter $\alpha$, and let $M(B), M(C)$ be the corresponding string modules. Then there exists a (canonical) morphism $f$ : $M(C) \rightarrow M(B)$ such that $f\left(z_{0}\right)=z_{0}$. Moreover, every such morphism is proper, meaning that there is no morphism $g: M(B) \rightarrow M(C)$ such that $g\left(z_{0}\right)=z_{0}$.

Proof. This is just a graph map in the sense of 3. Let us include the description for completeness.

Note that for every string $B=b_{1} \ldots b_{n}$ there is a canonical embedding $M(B) \rightarrow$ $M(B \beta D)$ given by $z_{i} \rightarrow z_{i}, i \leq n$, and also a canonical projection $M\left(B \gamma^{-1} E\right) \rightarrow$ $M(B)$ given by $z_{i} \rightarrow z_{i}, i \leq n$ and $z_{j} \rightarrow 0$ for $j>n$.

Now, if $B<C$ by 1 ), then define $f: M(C) \rightarrow M(B)$ to be the projection $M(C)=M\left(B \gamma^{-1} D\right) \rightarrow M(B)$. If $B<C$ by 2$)$, then define $f: M(C) \rightarrow M(B)$ to be the embedding $M(C) \rightarrow M(C \beta E)=M(B)$. For 3) we obtain $f$ as a composite $\operatorname{map} M(C)=M\left(B^{\prime} \gamma^{-1} G\right) \rightarrow M\left(B^{\prime}\right) \rightarrow M\left(B^{\prime} \beta F\right)=M(B)$.

Suppose that there exists a map $g: M(B) \rightarrow M(C)$ such that $g\left(z_{0}\right)=z_{0}$. Note that $M(C)$ is indecomposable and pure-injective (being of finite dimension). By [6, Prop. 4.26], every noninvertible endomorphism of $M(C)$ strongly increases the pp-type of every nonzero element. Since $g f\left(z_{0}\right)=z_{0}$, it follows that $g f$ is invertible. Therefore, $M(B)=\operatorname{im}(f) \oplus \operatorname{ker}(g)$. Since $M(B)$ is indecomposable, and $f, g \neq 0$, we conclude that $f$ is epi and $g$ is mono. By symmetry, $f$ is mono and $g$ is epi; hence $M(C) \cong M(B)$, a contradiction.

Since $M(C)$ is finitely presented, by [6, Prop. 8.4] there is a (positive primitive) formula $\varphi_{C}(x)$ which generates the pp-type of $z_{0}$ in $M(C)$. 
Corollary 2.2. Let $B<C$ be strings with first letter $\alpha$. Then $\varphi_{B} \rightarrow \varphi_{C}$, and this implication is proper.

Proof. Let $p$ be the pp-type of $z_{0}$ in $M(B)$, and let $q$ be the pp-type of $z_{0}$ in $M(C)$. Then $p$ is generated by $\varphi_{B}$, and $q$ is generated by $\varphi_{C}$. By Lemma 2.1 there is a morphism $f: M(C) \rightarrow M(B)$ such that $f\left(z_{0}\right)=z_{0}$. It follows that $q \subseteq p$; hence $\varphi_{B} \rightarrow \varphi_{C}$. Suppose that $\varphi_{C} \rightarrow \varphi_{B}$. Then there exists a morphism $g: M(B) \rightarrow M(C)$ such that $g\left(z_{0}\right)=z_{0}$, which contradicts Lemma 2.1.

Clearly $\varphi_{C}$ can be chosen of the form $\exists z_{1}, \ldots, z_{n}$, followed by a complete description of the action on $z_{i}$. In fact, for $1 \leq i \leq n-1$ it suffices to describe only the action given by $c_{i}$ and $c_{i+1}$. Indeed, suppose we have $\alpha^{-1} z_{i} \beta$ in $M(C)$, i.e., $\alpha$ and $\beta$ are different arrows ending in $z_{i}$. Then, for every arrow $\gamma$ starting in $z_{i}$, we have either $\gamma \alpha=0$ or $\gamma \beta=0$; hence $\gamma z_{i}$ must be zero. If $c_{1}=\alpha$ is a direct arrow, and there exists $\gamma$ such that $\gamma \alpha \neq 0$, then the formula should, in addition, say $\gamma z_{0}=0$. Similarly, we may need to add one more relation for $z_{n}$.

\section{WIDTH}

Let $L_{1}$ and $L_{2}$ be chains with 0 and 1 . By $L=L_{1} \otimes L_{2}$ we will denote the modular lattice freely generated by $L_{1}$ and $L_{2}$. It is well known (see [4, Th. 13]) that this lattice is distributive. Moreover, it is quite easy to visualize the structure of $L$. Let $L_{1} \times L_{2}$ be presented as a plane. We assign to $a \in L_{1}$ the vertical strip $[0 ; a] \times L_{2}$, and to $b \in L_{2}$ the horizontal strip $L_{1} \times[0 ; b]$ :
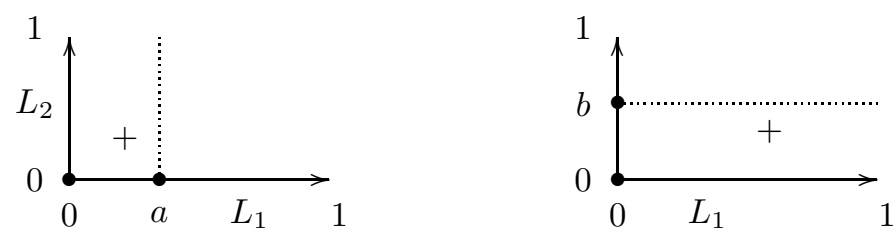

It follows from [4 proof of Th. 13] that the lattice generated by these strips (with respect to ordinary meet and joint operations) is isomorphic to $L$. To be more precise, we should avoid $1 \in L_{1}$ and $1 \in L_{2}$ being glued together via this representation. This can be overcome as follows: add formally $\infty$ after 1 in $L_{1}$, and the same for $L_{2}$.

Note that an element $a \wedge b$ is represented as a rectangle $[0 ; a] \times[0 ; b]$. Since $L$ is distributive, and $L_{1}, L_{2}$ are chains, every element $l \in L$ can be written as $l=\left(a_{n} \wedge b_{1}\right)+\left(a_{n-1} \wedge b_{2}\right)+\cdots+\left(a_{1} \wedge b_{n}\right)$, where $a_{1}<\cdots<a_{n} \in L_{1}$ and $b_{1}<\cdots<b_{n} \in L_{2}$ (we use + instead of $\vee$ ). Thus a typical element of $L$ looks like a descending ladder (where the first step may be of infinite height and the last of infinite length):

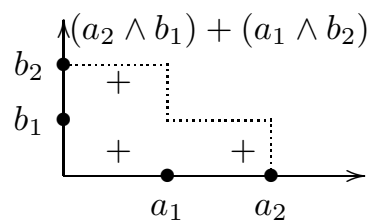

Note that we can rearrange brackets in $l: l=a_{n} \wedge\left(b_{1}+a_{n-1}\right) \wedge \cdots \wedge\left(b_{n-1}+a_{1}\right) \wedge b_{n}$. Given $a, b \in L,(a / b)$ will denote the interval $[a \wedge b ; a]$. 
Let $a<a^{\prime} \in L_{1}, b<b^{\prime} \in L_{2}$. Then the interval $\left(a^{\prime} \wedge b^{\prime} / a+b\right)=\left[a^{\prime} \wedge b^{\prime} \wedge(a+\right.$ $\left.b) ; a^{\prime} \wedge b^{\prime}\right]=\left[\left(a \wedge b^{\prime}\right)+\left(a^{\prime} \wedge b\right) ; a^{\prime} \wedge b^{\prime}\right]$ can be thought as the set-theoretical difference of the representing figures, i.e., as a rectangle $P=\left[a ; a^{\prime}\right] \times\left[b ; b^{\prime}\right]$ (caution: $P$ is not an element of $L$ ):

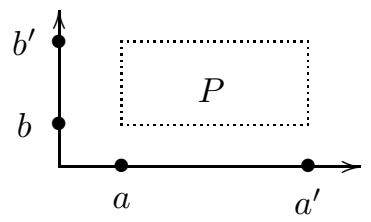

Given a lattice $L$ with 0 and 1 , we define the 2 -dimension of $L, 2 \operatorname{dim}(L)$, by induction on the ordinals. Put $2 \operatorname{dim}(L)=0$ iff $0=1$ in $L$, i.e., $L$ consists of one element. Set $2 \operatorname{dim}(L)=\alpha$ if $2 \operatorname{dim}(L)$ is not less than $\alpha$, and for every $a \in L$ we have either $2 \operatorname{dim}(a / 0)<\alpha$ or $2 \operatorname{dim}(1 / a)<\alpha$. For instance, $2 \operatorname{dim}\left(2^{n}+1\right)=n+1$ and $2 \operatorname{dim}(\omega+1)=\omega$. Thus $2 \operatorname{dim}(L)$ shows how many times we can split $L$ into two "equal" pieces. Note that $2 \operatorname{dim}(L)$ is defined iff $L$ does not contain the rationals as a suborder.

Let $L$ be a (modular) lattice with 0 and 1 . The width of $L, \mathrm{w}(L)$, will be defined by induction on the ordinals. Set $\mathrm{w}(L)=0$ iff $0=1$ in $L$. Now put $\mathrm{w}(L)=\alpha$, if $\mathrm{w}(L)$ is not less than $\alpha$, and for every $a, b \in L$ we have either $(a+b / a)<\alpha$ or $(a+b / b)<\alpha$. For instance, if $L$ is a chain with at least two elements, then $\mathrm{w}(L)=1$. The width of $L$ estimates how many "diamonds" can be repeatedly embedded in $L$. In particular, $\mathrm{w}(L) \geq \alpha+1$ if there are $a, b \in L$ such that " $\alpha$ diamonds" can be nested in each of the intervals $(a+b / a)$ and $(a+b / b)$, hence also in $(a / a \wedge b)$ and $(b / a \wedge b)$ :

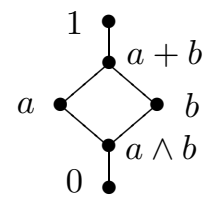

Proposition 3.1. Let $L_{1}$ and $L_{2}$ be chains with 0 and 1 such that $2 \operatorname{dim}\left(L_{1}\right)=\alpha$, $\operatorname{dim}\left(L_{2}\right)=\beta$, and let $L=L_{1} \otimes L_{2}$. Then $\mathrm{w}(L) \geq \min (\alpha, \beta)$.

Proof. Let $a<a^{\prime} \in L_{1}, b<b^{\prime} \in L_{2}$ be such that $2 \operatorname{dim}\left(a^{\prime} / a\right) \geq \alpha, 2 \operatorname{dim}\left(b^{\prime} / b\right) \geq \beta$ and $\alpha, \beta \geq \gamma$. By induction on $\gamma$ we prove that the width of the interval $\left(a^{\prime} \wedge b^{\prime} / a+b\right)$ (it looks like the rectangle $P=\left[a ; a^{\prime}\right] \times\left[b ; b^{\prime}\right]$ in the figure below) is not less than $\gamma$.

The proof is obvious when $\gamma$ is a limit. So let $\gamma=\delta+1$. Since $2 \operatorname{dim}\left(a^{\prime} / a\right) \geq \gamma$, there exists a $a^{\prime \prime} \in\left(a^{\prime} / a\right)$ such that $2 \operatorname{dim}\left(a^{\prime \prime} / a\right) \geq \delta$ and $2 \operatorname{dim}\left(a^{\prime} / a^{\prime \prime}\right) \geq \delta$. Similarly there exists a $b^{\prime \prime} \in\left(b^{\prime} / b\right)$ such that $2 \operatorname{dim}\left(b^{\prime \prime} / b\right) \geq \delta$ and $2 \operatorname{dim}\left(b^{\prime} / b^{\prime \prime}\right) \geq \delta$ :

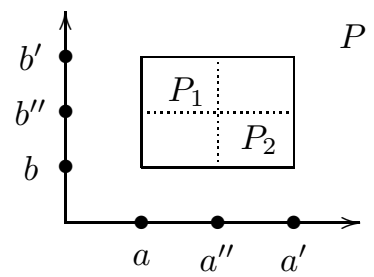

We prove that there are $\theta_{1}, \theta_{2} \in L$ such that

1) $\left(a^{\prime} \wedge b^{\prime}\right) \wedge(a+b)<\theta_{1}, \theta_{2}<a^{\prime} \wedge b^{\prime}$ 
2) the interval $\left(\theta_{1}+\theta_{2} / \theta_{1}\right)$ is isomorphic to the interval $\left(a^{\prime \prime} \wedge b^{\prime} / a+b^{\prime \prime}\right)=$ $\left(a^{\prime \prime} \wedge b^{\prime}\right) /\left(a^{\prime \prime} \wedge b^{\prime \prime}\right)+\left(a \wedge b^{\prime}\right)$ (it looks like the rectangle $P_{1}=\left(a^{\prime \prime} / a\right) \times\left(b^{\prime} / b^{\prime \prime}\right)$ on the figure);

3) the interval $\left(\theta_{1}+\theta_{2} / \theta_{2}\right)$ is isomorphic to the interval $\left(a^{\prime} \wedge b^{\prime \prime} / a^{\prime \prime}+b\right)=$ $\left(a^{\prime} \wedge b^{\prime \prime}\right) /\left(a^{\prime} \wedge b\right)+\left(a^{\prime \prime} \wedge b^{\prime \prime}\right)$ (it looks like the rectangle $P_{2}=\left(a^{\prime} / a^{\prime \prime}\right) \times\left(b^{\prime \prime} / b\right)$ on the figure).

Then, by the induction hypothesis, we would have $\mathrm{w}\left(P_{1}\right) \geq \delta$, $\mathrm{w}\left(P_{2}\right) \geq \delta$; therefore $\mathrm{w}(P) \geq \gamma$.

It is quite easy to prove this on the level of figures (just insert $P_{1}$ and $P_{2}$ in $P$ ), but the lattice-theoretical proof is comparatively harder.

Take $\theta_{1}=\left(a^{\prime} \wedge b^{\prime \prime}\right)+\left(a \wedge b^{\prime}\right)=a^{\prime} \wedge\left(b^{\prime \prime}+a\right) \wedge b^{\prime}$ and $\theta_{2}=\left(a^{\prime} \wedge b\right)+\left(a^{\prime \prime} \wedge b^{\prime}\right)=$ $a^{\prime} \wedge\left(b+a^{\prime \prime}\right) \wedge b^{\prime}:$
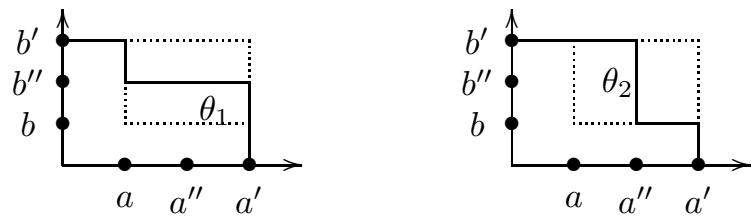

In particular, $\theta_{1}$ and $\theta_{2}$ are incomparable, and $a^{\prime} \wedge b^{\prime} \wedge(a+b)<\theta_{1}, \theta_{2}<a^{\prime} \wedge b^{\prime}$. Clearly we have $\theta_{1}+\theta_{2}=\left(a^{\prime} \wedge b^{\prime \prime}\right)+\left(a^{\prime \prime} \wedge b^{\prime}\right)=a^{\prime} \wedge\left(b^{\prime \prime}+a^{\prime \prime}\right) \wedge b^{\prime}$ :

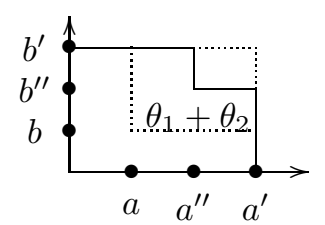

To prove 2), take $\varphi_{1}=a^{\prime \prime} \wedge b^{\prime}$ and $\psi_{1}=\left(a^{\prime \prime} \wedge b^{\prime \prime}\right)+\left(a \wedge b^{\prime}\right)$ :
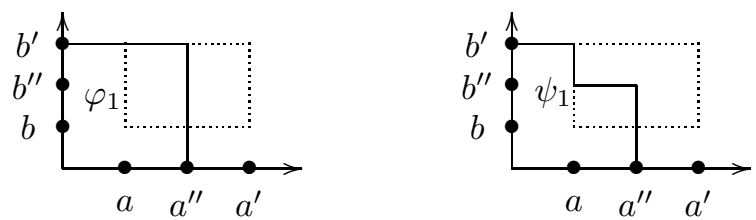

Then $\psi_{1}<\varphi_{1}$, and $\left(\varphi_{1} / \psi_{1}\right)$ is a rectangle $P_{1}$. To prove that $\left(\varphi_{1} / \psi_{1}\right) \cong\left(\theta_{1}+\right.$ $\left.\theta_{2} / \theta_{1}\right)$ it suffices to check that $\varphi_{1}+\theta_{1}=\theta_{1}+\theta_{2}$ and $\varphi_{1} \wedge \theta_{1}=\psi_{1}$.

But this is easily seen from the above figures.

To prove 3), let $\varphi_{2}=a^{\prime} \wedge b^{\prime \prime}$ and $\psi_{2}=\left(a^{\prime} \wedge b\right)+\left(a^{\prime \prime} \wedge b^{\prime \prime}\right)$ :
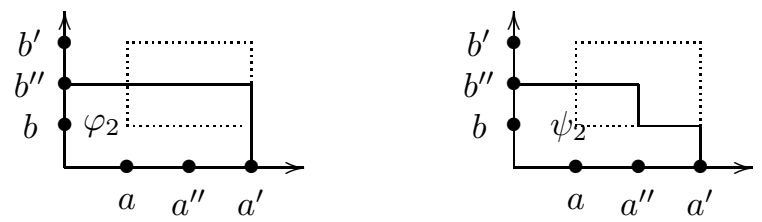

Then $\psi_{2}<\varphi_{2}$, and $\left(\varphi_{2} / \psi_{2}\right)$ is a rectangle $P_{2}$.

To prove that $\left(\varphi_{2} / \psi_{2}\right) \cong\left(\theta_{1}+\theta_{2} / \theta_{2}\right)$, it suffices to check that $\varphi_{2}+\theta_{2}=\theta_{1}+\theta_{2}$ and $\varphi_{2} \wedge \theta_{2}=\psi_{2}$. This is also evident from the above figures. 
Corollary 3.2. Let $L_{1}$ and $L_{2}$ be lattices with 0 and 1 such that both $2 \operatorname{dim}\left(L_{1}\right)$ and $2 \operatorname{dim}\left(L_{2}\right)$ are undefined. Then $\mathrm{w}\left(L_{1} \otimes L_{2}\right)$ is undefined.

\section{THE MAIN RESUlT}

We are in a position to prove the main result of the paper.

Theorem 4.1. Let $A$ be a non-domestic string algebra. Then the width of the lattice of all pp-formulae over $A$ is equal to infinity.

Proof. Recall that a band is a string $C=c_{1} \ldots c_{n}$ of length $\geq 2$ such that 1) all powers $C^{m}$ are defined; 2) $C$ is not a power of a string of smaller length; 3) $c_{1}$ is a direct letter and $c_{n}$ is an inverse letter. If $c_{1}=\alpha$ and $c_{n}=\beta^{-1}$, then 1) yields that $\alpha$ and $\beta$ are different arrows ending in the same vertex. Thus $\beta$ is uniquely determined by $\alpha$.

Following [11], for an arrow $\alpha$, by $B(\alpha)$ we denote the set of all bands with first letter $\alpha$. By what we have said above, the last letter $\beta^{-1}$ is the same for all bands in $B(\alpha)$. Since $A$ is not domestic, by [11, p. 41] there is an $\alpha$ for which there are two different bands $B, C \in B(\alpha)$, such that $B$ and $C$ contain no substring of the form $\beta^{-1} \alpha$.

Let $B=b_{1} \ldots b_{n}$ and $C=c_{1} \ldots c_{m}$, where we may assume that $B<C$. Note that the case 2) (see the definition of $<$ above) is not possible. Indeed, then $B=C \delta E$; hence $\delta=\alpha$, and $B$ contains a substring $\beta^{-1} \alpha$, a contradiction. Thus either 1) $C=B \gamma^{-1} D$ or 3$) B=B^{\prime} \delta F$ and $C=B^{\prime} \gamma^{-1} G$. In both cases $B C<C T$ for all $T$. Indeed, this is clear for 3) (see remark above). For 1 ) it is also clear, since $B C=B \alpha C^{\prime}$ and $C T=B \gamma^{-1} D T$.

Thus (see also [7, p. 450]) we obtain the following strongly descending chain of strings (every word consisting of the letters $B$ and $C$ is a string):

$$
B C>B C B C>B C B^{2} C>B C B^{2} C B C>B C B^{2} C B^{2} C>B^{2} C .
$$

The interval $\left[B^{2} C ; B C\right]$ is isomorphic to $\left[B C B^{2} C ; B C B C\right]$ (after removing $B C$ from the beginning) and $\left[B C B^{2} C B^{2} C ; B C B^{2} C B C\right]$ (after removing $B C B^{2} C$ from the beginning). Thus we may extend this construction to obtain a dense subchain $L_{1}$ of $<$ with 0 and 1 .

Recall that for each $T \in L_{1}$ we have defined a pp-formula $\varphi_{T}(x)$. By Lemma 2.2 for all $T<U \in L_{1}$ we have $\varphi_{T} \rightarrow \varphi_{U}$, and this implication is proper. Thus we obtain a dense chain of pp-formulae over $A$ (we will use $L_{1}$ also to denote this chain).

Now let us consider the words $B^{-1}$ and $C^{-1}$. The first letter of both words is $\beta$, the last letter is $\alpha^{-1}$ and both $B$ and $C$ contain no substring $\alpha^{-1} \beta$. Thus we may repeat our constructions to obtain a densely ordered chain $L_{2}$ (with 0 and 1 ) of words constructed from the letters $B^{-1}$ and $C^{-1}$. Note that every word in $L_{2}$ has $\beta$ as a starting arrow and $\beta \neq \alpha$. As above we may consider $L_{2}$ as a densely ordered chain of pp-formulae $\psi_{S}$ such that $\psi_{S} \rightarrow \psi_{T}$ iff $S<T$.

For every $V \in L_{2}, U \in L_{1}$ we may consider a string module $M=M\left(V^{-1} U\right)$ defined by the word $V^{-1} U$ with the basis $z_{-m}, \ldots, z_{0}, z_{1}, \ldots, z_{n}$, such that $z_{0}$ is located between $V^{-1}$ and $U$ (in particular, $\beta z_{-1}=z_{0}$ and $\alpha z_{1}=z_{0}$ ). It is easy to check that $\left(M, z_{0}\right)$ is a free realization of $\varphi_{U} \wedge \psi_{V}$ (it is just the amalgam of modules $M(U)$ and $M(V)$ by the submodule generated by $z_{0}$; the other way to see this is to construct for every element $n$ of a module $N$ with $N \models\left(\varphi_{U} \wedge \psi_{V}\right)(n)$ a morphism $f: M \rightarrow N$ such that $\left.f\left(z_{0}\right)=n\right)$. 
Let $L$ be generated by $L_{1}$ and $L_{2}$ in the lattice of all pp-formulae over $A$. We prove that $L$ does not have width (then the lattice of all pp-formulae over $A$ does not have width). By Lemma 3.1, it suffices to check that $L$ is freely generated by $L_{1}$ and $L_{2}$. Considering the canonical forms of elements in $L$, it remains to prove the following: if $T<U \in L_{1}$ and $S<V \in L_{2}$, then $\varphi_{U} \wedge \psi_{V}$ does not imply $\varphi_{T}+\psi_{S}$.

Assume to the contrary that $\varphi_{U} \wedge \psi_{V}$ implies $\varphi_{T}+\psi_{S}$. Let $M=M\left(V^{-1} U\right), z_{0}$ be chosen (as above) to be a free realization of $\varphi_{U} \wedge \psi_{V}$. It is clear (see a similar proof in 8, p. 26]) that $\varphi_{T}(M)$ and $\psi_{S}(M)$ are homogeneous spaces, i.e., $\sum_{i} \lambda_{i} z_{i} \in \varphi_{T}(M)$ iff $z_{i} \in \varphi_{T}(M)$ for every $i$ with $\lambda_{i} \neq 0$. Thus $z_{0} \in \varphi_{T}(M)$ or $z_{0} \in \psi_{S}(M)$ yields that either $\varphi_{U} \wedge \psi_{V} \rightarrow \varphi_{T}$ or $\varphi_{U} \wedge \psi_{V} \rightarrow \psi_{S}$.

If $\varphi_{U} \wedge \psi_{V} \rightarrow \psi_{T}$, then there is a morphism $f: M \rightarrow M(T)$ such that $f\left(z_{0}\right)=z_{0}$. Restricting $f$ to the submodule $M(U)$, we obtain a contradiction to Lemma 2.1 Similarly $\varphi_{U} \wedge \psi_{V} \rightarrow \psi_{S}$ yields (since $M(V)$ is a submodule of $M$ ) $\psi_{V} \rightarrow \psi_{S}$, a contradiction again.

Corollary 4.2. Let $A$ be a (finite-dimensional) non-domestic string algebra over a countable field. Then there exists a superdecomposable pure-injective A-module.

Proof. By Theorem 4.1 the width of the lattice of all pp-formulae over $A$ is undefined. It remains to apply [6, Thm. 10.13].

\section{ACKNOWLEDGMENT}

I am indebted to Mike Prest for his helpful suggestions and interest.

\section{REFERENCES}

[1] S. Baratella and M. Prest, Pure-injective modules over the dihedral algebras, Comm. Algebra, 25(1) (1997), 11-31. MR 98d:16021

[2] M. Butler and C. M. Ringel, Auslander-Reiten sequences with few middle terms and applications to string algebras, Comm. Algebra, 15 (1-2) (1987), 145-179. MR 88a:16055

[3] W. W. Crawley-Boevey, Maps between representations of zero-relation algebras, J. Algebra, 126 (1989), 259-263. MR 90k:16035

[4] G. Grätzer, General Lattice Theory, Pure and Applied Mathematics, Vol. 75, Academic Press, New York, 1978. MR 80c:06001b

[5] C. U. Jensen and H. Lenzing, Model-theoretic algebra with particular emphasis on fields, rings, modules, Algebra, Logic and Applications, Vol. 2, Gordon and Breach Science Publishers, New York, 1989. MR 91m:03038

[6] M. Prest, Model Theory and Modules, London Math. Soc. Lecture Note Series, Vol. 130, Cambridge University Press, Cambridge, 1988. MR 89h:03061

[7] M. Prest, Morphisms between finitely presented modules and infinite-dimensional representations, Canadian Math. Soc., Conference Proceedings, 24 (1998), pp. 447-455. MR 99i:16027

[8] C. M. Ringel, The indecomposable representations of the dihedral 2-groups, Math. Ann., 214 (1975), 19-34. MR 51:680

[9] C. M. Ringel, Some algebraically compact modules. I, Abelian Groups and Modules, A. Facchini and C. Menini, eds., Kluwer, Dordrecht, 1995, pp. 419-439. MR 97c:16016

[10] C. M. Ringel, Infinite length modules. Some examples as introduction, Infinite Length Modules, H. Krause and C. M. Ringel, eds., Trends in Math., Birkhäuser, Basel, 2000, pp. 1-73. MR 2002d: 16002

[11] J. Schröer, Hammocks for string algebras, Doctoral thesis, 1997.

[12] M. Ziegler, Model theory of modules, Annals Pure Appl. Logic, 26 (1984), 149-213. MR 86c:03034

Department of Mathematics, The Ohio State University at Lima, 4240, Campus Drive, LimA, ОНIо 45804

E-mail address: puninskiy.1@osu.edu 